\title{
Successful and Safe Implementation of a Trinary Interpretation and Reporting Strategy for V/Q Lung Scintigraphy
}

\author{
Joseph E. Glaser ${ }^{1}$, Murthy Chamarthy ${ }^{1,2}$, Linda B. Haramati ${ }^{3,4}$, David Esses ${ }^{5}$, and Leonard M. Freeman ${ }^{1,3}$ \\ ${ }^{I}$ Department of Nuclear Medicine, Albert Einstein College of Medicine, Montefiore Medical Center, Bronx, New York; ${ }^{2}$ Department of \\ Radiology, Bridgeport Hospital, Bridgeport, Connecticut; ${ }^{3}$ Department of Radiology, Albert Einstein College of Medicine, Montefiore \\ Medical Center, Bronx, New York; ${ }^{4}$ Department of Medicine, Montefiore Medical Center, Bronx, New York; and ${ }^{5}$ Department of \\ Emergency Medicine, Montefiore Medical Center, Bronx, New York
}

For the past 4 decades, ventilation-perfusion (V/Q) scan interpretation for pulmonary embolism (PE) was performed using probability-based assessments, which were neither wellreceived nor well-understood by many clinicians. Recently, we combined normal, very low probability, and low-probability interpretations in emergency department patients and found a false-negative $(\mathrm{FN})$ rate of $1.2 \%$ on follow-up. Afterward, we transitioned to a new trinary interpretative strategy: no PE, PE present, and nondiagnostic. In this series, we compared the outcomes of the traditional and trinary interpretative strategies. Methods: We retrospectively identified all patients undergoing V/Q scans for the 1 year straddling the shift in interpretive strategy, with traditional interpretation being used between September 18, 2008, and March 17, 2009, and trinary interpretation being used between March 18, 2009, and September 17, 2009. A FN study was defined as development of deep vein thrombosis or PE within 3 months after a negative baseline evaluation. Results: The traditional interpretation group included 208 male patients (27\%) and 570 female patients $(73 \%)$, with a mean age $( \pm S D)$ of $50.9 \pm 18.4$ years. These interpretations $(n=778)$ were high probability in $4.9 \%(38)$, intermediate probability in 5\% (39), low probability in $59.5 \%$ (463), very low probability in $17.2 \%$ (134), and normal in $13.4 \%$ (104). The trinary interpretation group included 181 male patients $(27 \%)$ and 483 female patients $(73 \%)$, with a mean age of $50.0 \pm 18.5$ years. These interpretations (664) were positive in $8.4 \%$ (56), negative in $88.1 \%(585)$, and nondiagnostic in $3.5 \%$ (23). The FN rate was $1.14 \%(8 / 701 ; 7$ deep vein thrombosis and $1 \mathrm{PE}$ ) for pooled normal, very low probability, and low probability in traditional interpretations versus $1.5 \%(9 / 585,5$ deep vein thrombosis and $4 \mathrm{PE})$ in trinary interpretations $(P=$ 0.63). The individual FN rates for the normal, very low probability, and low-probability groups were $0.0 \%, 0.75 \%$, and $1.51 \%$, respectively ( $P=0.36$ for normal vs. low probability). Pediatric subgroup analysis showed 19 traditional interpretations: $5.3 \%$ high (1); 0 intermediate; and $94.7 \%$ (18) low probability, very low probability, and normal. 20 trinary interpretations were positive in $10 \%(2)$, nondiagnostic in $5 \%(1)$, and

\footnotetext{
Received Mar. 18, 2011; revision accepted May 4, 2011.

For correspondence or reprints contact: Leonard M. Freeman, Department of Nuclear Medicine, Montefiore Medical Center, 111 E. 210th St., Bronx, NY, 10467.

E-mail: Ifreeman@montefiore.org.

Published online Jul. 29, 2011.

COPYRIGHT @ 2011 by the Society of Nuclear Medicine, Inc.
}

negative in $85 \%$ (17), with no FNs using either strategy. Conclusion: A simplified trinary interpretation strategy for V/Q lung scintigraphy provides outcomes similar to traditional probability assessments and facilitates clear communication.

Key Words: pulmonary embolism; V/Q; lung scintigraphy; lung scan

J Nucl Med 2011; 52:1508-1512

DOI: 10.2967/jnumed.111.090753

$\mathbf{O}$ ver the past several decades, there has been a huge increase in patient radiation exposure from medical imaging, with a large portion of this increase attributed to CT $(1,2)$, Not only has this issue received attention in the medical literature $(2,3)$, but the general media (4) has recently publicized several incidents of radiation toxicity from imaging and therapy, bringing this issue to public awareness. Governmental agencies have taken notice and are responding $(5,6)$, and physicians are being urged to safely reduce patient radiation exposure.

Lung ventilation-perfusion (V/Q) scintigraphy was the imaging method of choice for suspected pulmonary embolism (PE) until the early 1990s. As CT pulmonary angiography (CTPA) developed, it became the dominant imaging modality for suspected PE, largely supplanting V/Q scanning in the United States by $2001(7,8)$. The effective radiation dose from CTPA is approximately 5 times that from V/Q scanning, with a 20-40 times higher dose to the female breast $(9,10)$. Many physicians are not aware of these differences $(11,12)$.

Traditional V/Q scan interpretations using probabilitybased reports have always been poorly understood and confusing to referring clinicians (13). There is a significant difference in the range of the likelihood of PE among interpreting physicians (14). Clinician preference for CTPA is related to greater availability, shorter scan times, and more clearly stated results. Although CTPA reporting has traditionally been trinary (negative, positive, and nondiagnostic), a patient's pretest probability similarly affects the 
predictive values and likelihood of having PE for both CTPA and V/Q scans (15-17), supporting the view that $\mathrm{V} / \mathrm{Q}$ results can be reported using terminology similar to that of CTPA.

Our institution is focused on a collaborative approach to reducing patient radiation exposure. We recently successfully changed practice patterns by working with the Emergency Department to establish the use of chest radiography to triage between the two studies. This change led to decreased radiation exposure by safely increasing the use of V/Q scanning and decreasing the use of CTPA. Patient outcomes were unaffected (18).

We subsequently implemented a trinary interpretative system for V/Q scans in clinical practice on March 18, 2009, similar to the interpretative strategy for CTPA. V/Q scans were interpreted as "PE present," "PE absent," or "nondiagnostic." The present study was designed to evaluate the safety and accuracy of implementing this trinary V/Q scan interpretation system in a large urban academic medical center performing a large number of lung scintigraphy studies.

\section{MATERIALS AND METHODS}

This study was approved by our institutional review board; informed consent was not required. In accordance with our usual clinical care, patients with normal results on chest radiography were referred for V/Q scans, as were patients with contraindications to CTPA regardless of chest radiographic findings. This algorithm was generally well accepted, as clinicians at our institution traditionally have had a high comfort level with V/Q scanning.

V/Q lung scans are available 24 hours a day, 7 days a week at our institution (Montefiore Medical Center, Bronx, NY). Ventilation imaging was performed routinely with $1,480 \mathrm{MBq}(40 \mathrm{mCi})$ of aerosolized ${ }^{99 \mathrm{~m} T c}$-labeled diethylenetriaminepentaacetic acid (Aerovent; MediNuclear, or Swirler; Amici) followed by intravenous administration of $148 \mathrm{MBq}$ (4 mCi) of ${ }^{99 \mathrm{~m}}$ Tc-labeled macroaggregated albumin for the perfusion study. Both ventilation and perfusion images were acquired in 8 standard projections for 100 seconds using a photopeak of $140 \mathrm{keV}$ (20\% window) on a SkyLight or Forte ADAC gamma camera (Philips Healthcare) to obtain approximately 100,000 and 500,000 counts, respectively. During the regular workday, V/Q scans were interpreted by experienced board-certified nuclear medicine physicians. Radiology residents (postgraduate year 3-5) interpreted the examinations that were performed during off-hours. All our residents receive extensive training in V/Q scan interpretations. A technologist is present in the hospital from 8:00 AM until midnight on weekdays and from 8 AM until 4 PM on Saturdays. Otherwise, the technologist is on call to the hospital and usually arrives within 30 minutes to perform a study. Final reports of all V/Q scans were reviewed for this study. Agreement between the residents' preliminary reports and the final report is consistently high in our institution, with amended reports issued in less than $1 \%$ of cases (18).

Our study population comprised inpatients, outpatients, and Emergency Department patients who underwent V/Q scanning from September 18, 2008, through September 17, 2009. This interval encompasses the 6 months before and the 6 months after clinical implementation of the trinary interpretative strategy for V/Q scanning on March 18, 2009 (Table 1). The cases were iden-
TABLE 1

Patient Demographics

\begin{tabular}{lcc}
\hline \multicolumn{1}{c}{ Demographic } & $\begin{array}{c}\text { Traditional } \\
\text { interpretation }\end{array}$ & $\begin{array}{c}\text { Trinary } \\
\text { interpretation }\end{array}$ \\
\hline Male $(n)$ & $208(27 \%)$ & $181(27 \%)$ \\
Female $(n)$ & $570(73 \%)$ & $483(73 \%)$ \\
$\begin{array}{c}\text { Mean age } \pm \text { SD } \\
\text { (years) }\end{array}$ & $50.9 \pm 18.4$ & $50.0 \pm 18.5$ \\
\hline
\end{tabular}

tified by review of patient examination records in the Nuclear Medicine Department. Patients with incomplete examinations (i.e., underwent ventilation or perfusion study only) were excluded.

The traditional interpretative strategy was in use during the first half of the study period (September 18, 2008, through March 17, 2009), and the trinary strategy was used during the second half of the study period (March 18, 2009, through September 18, 2009). This change was initiated after direct discussions with the Radiology Department and the Emergency Medicine Department and the formal issuance of a memorandum by the Nuclear Medicine Department director (Fig. 1). The data were then categorized by interpretive strategy. Traditional criteria included normal, very low probability, low-probability, intermediate-probability, and highprobability categories. The trinary scheme categorized the scans as negative, positive, or nondiagnostic.

Starting with the change to trinary interpretation, we began to consider single-segmental mismatches to be positive for PE. Although the original PIOPED I criteria had interpreted these single-segmental mismatches as low probability, the subsequent 1993 modified PIOPED report had placed them in the intermediate-probability category (19). This group of patients was now further upgraded in our study to a positive diagnostic category. This decision was consistent with prior work by Stein et al. (20) showing that patients with V/Q mismatches of $>1$ and no underlying cardiopulmonary disease can be further upgraded from the modified PIOPED I intermediate category to high probability with a positive predictive value of $86 \%$. We considered negative chest radiography results to be equivalent to no significant underlying cardiopulmonary disease.

For every patient in the database, results of all V/Q and CT scans and duplex examinations of the lower extremities were recorded for 3 months after the index evaluation. No patients underwent traditional pulmonary angiography. Patient mortality was identified by chart review and the Social Security Death Index. Some patients underwent multiple studies ( $n=34$ patients) spanning both periods. However, each patient was counted only once, at initial presentation during the study period. Subsequent examinations for these patients were not used for the final tabulation but were used for follow-up, when appropriate.

Patients were categorized as baseline-negative if V/Q scans were interpreted as normal, very low probability, or low probability in the first half of the study period or PE-negative during the second half of the study period and the patients did not have deep vein thrombosis on baseline lower-extremity duplex examination. Examinations were considered to be falsely negative (FN) if new deep vein thrombosis or PE was diagnosed on follow-up V/Q scanning, CT, or duplex examination of the lower extremities within 3 months of follow-up (21). Differences between patients evaluated using the traditional and trinary interpretative strategies 
March 18, 2009

TO: $\quad$ All Radiology Residents and Attending Physicians All Nuclear Medicine Residents and Attending Physicians

Director, Moses Emergency Department

FROM: $\quad$ Leonard M. Freeman, M.D.

\section{SUBJECT: LUNG SCAN INTERPRETATIONS}

As we discussed, we will be instituting a significant change in the language used in reporting V/Q lung scans for suspected pulmonary embolism (PE). The intent is to abandon the use of frequently misunderstood "probability" interpretations. Instead we will use a simpler interpretive scheme as follows:

\section{Previous Interpretation}

Normal, very low and low probability

High probability

Intermediate/Indeterminate probability

\section{New Interpretation}

No evidence of PE

PE present

Non-Diagnostic study
FIGURE 1. Memorandum sent to Emergency Medicine Department, Radiology Department, and Nuclear Medicine Department on March 18, 2009, announcing change in policy and implementation of new reporting scheme.

For each of these interpretations, it must be understood that if there is discordance between this interpretation and objective clinical assessment, i.e., pre-test probability or Wells Score, further testing may be needed.

Additionally, to further clarify the clinical significance of "No evidence of PE" reports, it should be stated that "No Evidence of PE" interpretations are associated with a $<10 \%$ likelihood of PE, i.e. a $90 \%$ negative Predictive Value.

Please distribute this note to your entire staff so that they will be fully informed with this change which will, hopefully, provide them with easy to understand lung scan interpretations.

were compared using 2-tailed Fisher exact tests, as appropriate (QuickCalcs Online Calculators for Scientists; GraphPad Software, Inc. (22)).

\section{RESULTS}

The results of our data collection are summarized in Table 2. The traditional (probability-based) interpretation group included 778 examinations, with 38 (4.9\%) being high probability, $39(5 \%)$ intermediate probability, $463(59.3 \%)$ low probability, 134 (17.2\%) very low probability, and 104
(13.4\%) normal. The trinary interpretation group included 664 examinations, with $56(8.4 \%)$ being PE present, 23 (3.5\%) nondiagnostic, and $585(88.1 \%)$ PE negative. The FN rate (Table 3) was $1.14 \%$ (8/701) for the pooled traditional interpretation and $1.5 \%(9 / 585)$ for the trinary interpretation $(P=$ $0.63)$. There was a statistically significant difference between the proportion of high-probability interpretations $(4.9 \%)$ and PE-present interpretations $(8.4 \%)(P=0.0073)$ but no significant difference when intermediate-probability interpretations were compared with nondiagnostic interpretations $(P=0.18)$

TABLE 2

Comparison of Results Using the 2 Reporting Schemes

\begin{tabular}{|c|c|c|c|c|}
\hline \multicolumn{2}{|c|}{ Traditional interpretation $(n=778)$} & \multicolumn{2}{|c|}{ Trinary interpretation $(n=664)$} & \multirow[b]{2}{*}{$P$ (Fisher's 2-tailed test) } \\
\hline Finding & $n$ & Finding & $n$ & \\
\hline High probability & $38(4.9 \%)$ & PE present & $56(8.4 \%)$ & $0.0073^{\star}$ \\
\hline Intermediate & $39(5 \%)$ & Nondiagnostic & $23(3.5 \%)$ & 0.18 \\
\hline Low probability & $463(59.5 \%)$ & & & \\
\hline Very low probability & $134(17.2 \%)$ & PE absent & $585(88.1 \%)$ & 0.23 \\
\hline Normal & $104(13.4 \%)$ & & & \\
\hline $\begin{array}{l}\text { Pooled normal, low probability, } \\
\text { and very low probability }\end{array}$ & $701(90.1 \%)$ & & & \\
\hline *Statistically significant. & & & & \\
\hline
\end{tabular}


TABLE 3

Comparison of FN Rates Using the 2 Reporting Schemes

\begin{tabular}{|c|c|c|c|c|c|c|c|c|}
\hline \multicolumn{4}{|c|}{ Traditional interpretation } & \multicolumn{4}{|c|}{ Trinary interpretation } & \multirow[b]{2}{*}{$\begin{array}{c}P \text { (Fisher's } \\
\text { 2-tailed test) }\end{array}$} \\
\hline & $\begin{array}{c}n \text { per } \\
\text { category }\end{array}$ & FN cases & FN rate & & $\begin{array}{c}n \text { per } \\
\text { category }\end{array}$ & FN cases & FN rate & \\
\hline Low probability & 463 & 7 & $1.51 \%$ & $\begin{array}{c}\mathrm{PE} \\
\text { negative }\end{array}$ & 585 & $\begin{array}{l}9 \text { (5 deep vein } \\
\text { thrombosis, } 4 \text { PE) }\end{array}$ & $1.5 \%$ & 0.63 \\
\hline Very low probability & 134 & 1 & $0.75 \%$ & & & & & \\
\hline Normal & 104 & 0 & $0.0 \%$ & & & & & \\
\hline $\begin{array}{l}\text { Pooled normal, low } \\
\text { probability, and } \\
\text { very low probability }\end{array}$ & 701 & $\begin{array}{c}8 \text { (7 deep vein } \\
\text { thrombosis, } 1 \mathrm{PE} \text { ) }\end{array}$ & $1.14 \%$ & & & & & \\
\hline
\end{tabular}

or when pooled normal, very low probability, and lowprobability interpretations were compared with PE-absent interpretations $(P=0.23)$.

Further comparison was done on a subgroup (Table 4) of pediatric patients up to 21 years old. The traditional interpretation group included 19 examinations, with $1(5.3 \%)$ being high probability, 0 intermediate, $6(31.6 \%)$ low probability, $5(26.3 \%)$ very low probability, and $7(36.8 \%)$ normal. The trinary interpretation group included 20 examinations, with 2 (10\%) being PE present, 1 (5\%) nondiagnostic, and 17 (85\%) PE negative. No FNs were detected in either subgroup.

\section{DISCUSSION}

The probability-based language used for interpreting V/Q scans has been an historic barrier to its acceptance in clinical practice. We implemented an institutional change from traditional probability-based language to a straightforward trinary interpretative scheme in which the diagnosis of PE is either affirmed (PE present), refuted (PE absent) or the scan is deemed nondiagnostic. All normal, very low probability, and low-probability V/Q studies were grouped into the "negative" category. This choice was reasonable because the aggregate patient group with normal, very low probability, and low-probability V/Q scans had outcomes similar to the negative CTPA group, as was documented in previous work at our institution that showed comparable FN rates for CTPA $(1.1 \%)$ and V/Q scanning (1.2\%) (18). Studies previously categorized as "intermediate" became "nondiagnostic," whereas

TABLE 4

Comparison of the 2 Reporting Schemes in Pediatric and Adolescent Subgroup ( $\leq 21$ Years Old)

\begin{tabular}{ll}
\hline \multicolumn{1}{c}{$\begin{array}{c}\text { Traditional interpretation } \\
(n=19)\end{array}$} & \multicolumn{1}{c}{$\begin{array}{c}\text { Trinary interpretation } \\
(n=20)\end{array}$} \\
\hline Age range, 15-21 years & Age range, 19-21 years \\
High probability, 1 (5.3\%) & PE present, $2(10 \%)$ \\
Intermediate, 0 & Nondiagnostic, 1 (5\%) \\
Low probability, 6 (31.6\%) & \\
Very low probability, 5 (26.3\%) & PE negative, 17 (85\%) \\
Normal, 7 (36.8\%) & FNs, 0/20 $(0 \%)$ \\
FNs, 0/19 (0\%) & \\
\hline
\end{tabular}

positive studies remained unchanged as "PE present," with the exception of single-segmental perfusion defects. These were transferred from the intermediate/nondiagnostic to the high-probability/positive category. This easily understood analysis highlighted that a traditional, probability-based interpretative strategy for V/Q scanning was a barrier to effective communication and that report clarity could be enhanced by using a simplified, trinary system.

On the basis of our prior work (18), we hypothesized that this strategy would be safe and not increase the rate of FN examinations. We therefore compared the group of patients who underwent V/Q scanning during the 6 months before implementation of the trinary interpretative strategy with those who underwent V/Q scanning during the 6 months after implementation.

The FN rates (defined as a diagnosis of thromboembolic disease within 3 months after a negative baseline evaluation) for both groups was low, at $1.14 \%$ and $1.5 \%$, respectively, did not differ statistically from each another and were similar to those reported in the literature (21).

We found a significantly $(P=0.0073)$ higher number of positive cases and nonsignificant decreases in nondiagnostic $(P=0.18)$ and negative $(P=0.23)$ studies with the shift to the new system. Our more aggressive positive interpretation of the single-segmental mismatch significantly contributed to the greater number of positive studies after the change to the trinary interpretive scheme.

Another issue noted was a difference in the number of total examinations performed during the two 6-month periods. This difference was believed to be due mostly to a fluctuating tracer supply during part of the study period, as well as to seasonal differences in PE study referrals.

Limitations of the study include its retrospective nature, with the associated inherent biases. Interobserver agreement was not evaluated systematically. However, the attending staff physician agreed with the interpretation of the on-call resident $99 \%$ of the time. Referral physician interpretation of the report was not analyzed, although communications with the patients' clinicians were, anecdotally, clearer after the new reporting scheme was implemented. A more formal survey of referring physicians' understandings of and reactions to the new reporting scheme 
may be an area for further follow-up and study. Finally, SPECT images and computerized objective evaluation of lung scans were not performed, although such measures have been reported to improve accuracy (23).

A 2007 prospective Canadian study evaluated the negative predictive value of ${ }^{99 \mathrm{~m}} \mathrm{Tc}$-technegas macroaggregated albumin studies in 405 eligible patients and found it to be $98.5 \%$, with a FN rate of $1.48 \%$ (24). This result is comparable to our data, with similar outcomes. Although we believe that introducing SPECT (and SPECT/CT) imaging will improve results (25), debate still exists about the validity of reconstructing planar images from tomography (23) and the importance of clinical outcomes versus the accuracy of the diagnostic tests.

Although the triage strategy using chest radiography has obviously worked well for us, this strategy may be of more limited utility at locations that perform few V/Q scans and have imaging specialists who are less comfortable interpreting them. However, we hope that our work in this arena will continue to stimulate renewed interest in radionuclide lung scintigraphy as a valuable diagnostic tool $(26,27)$.

With the recent focus on patient radiation exposure $(2,3)$, any effort to reduce radiation-based examinations is to be encouraged. Our previous efforts (18) have shown that algorithms to reduce radiation exposure can be successfully implemented within a short time. The fact that our new paradigm for reporting results was safe and well accepted by the clinical services staff supports the notion that changes can be made that lead to improved communication between physicians. We hope that this change will improve patient care and enable less extensive testing to clarify confusing diagnoses.

\section{CONCLUSION}

Adopting a simplified trinary interpretation strategy for V/Q lung scintigraphy provides outcomes similar to standard probability assessments. There was no statistically significant difference in $\mathrm{FN}$ rates between the 2 reporting schemes $(P=0.63)$. Therefore, a trinary interpretative strategy can safely be implemented, facilitating clearer communication with referring clinicians.

\section{DISCLOSURE STATEMENT}

The costs of publication of this article were defrayed in part by the payment of page charges. Therefore, and solely to indicate this fact, this article is hereby marked "advertisement" in accordance with 18 USC section 1734.

\section{ACKNOWLEDGMENTS}

We acknowledge Louisa Olmo for her invaluable aid in preparing the patient databases upon which our study was based, and all the residents who helped put this strategy into practice. Dr. Glaser would like to thank his wife, Evelyn Glaser, CPNP, for her support during this project. No other potential conflict of interest relevant to this article was reported.

\section{REFERENCES}

1. Amis ES Jr, Butler PF, Applegate KE, et al. American College of Radiology white paper on radiation dose in medicine. J Am Coll Radiol. 2007;4:272-284.

2. Fazel R, Krumholz HM, Wang Y, et al. Exposure to low-dose ionizing radiation from medical imaging procedures. N Engl J Med. 2009;361:849-857.

3. Brenner DJ, Hricak H. Radiation exposure from medical imaging: time to regulate? JAMA. 2010;304:208-209.

4. Zarembo A. Cedars-Sinai radiation overdoses went unseen at several points. Los Angeles Times Web site. Available at: http://articles.latimes.com/2009/oct/14/ local/me-cedars-sinai14. Published October 14, 2009. Accessed July 19, 2011.

5. Paper W. Initiative to reduce unnecessary radiation exposure from medical imaging. U.S. Food and Drug Administration Web site. Available at: http://www.fda. gov/Radiation-EmittingProducts/RadiationSafety/RadiationDoseReduction/ ucm199994.htm. Updated December 14, 2011. Accessed July 11, 2011.

6. Bogdanich W, Ruiz RR. FDA to increase oversight of medical radiation. New York Times. February 9, 2010:A17.

7. Levin DC, Rao VM, Parker L, et al. Recent trends in utilization rates of noncardiac thoracic imaging: an example of how imaging growth might be controlled. J Am Coll Radiol. 2007;4:886-889.

8. Prologo JD, Gilkeson RC, Diaz M, et al. CT pulmonary angiography: a comparative analysis of the utilization patterns in emergency department and hospitalized patients between 1998 and 2003. AJR. 2004;183:1093-1096.

9. Einstein AJ, Henzlova MJ, Rajagopalan S. Estimating risk of cancer associated with radiation exposure from 64-slice computed tomography coronary angiography. JAMA. 2007;298:317-323.

10. Mettler FA Jr, Huda W, Yoshizumi TT, et al. Effective doses in radiology and diagnostic nuclear medicine: a catalog. Radiology. 2008;248:254-263.

11. Groves AM, Yates SJ, Win T, et al. CT pulmonary angiography versus ventilation-perfusion scintigraphy in pregnancy: implications from a UK survey of doctors' knowledge of radiation exposure. Radiology. 2006;240:765-770.

12. Shiralkar S, Rennie A, Snow M, et al. Doctors' knowledge of radiation exposure: questionnaire study. BMJ. 2003;327:371-372.

13. Gray HW, McKillop JH, Bessent RG. Lung scan reports: interpretation by clinicians. Nucl Med Commun. 1993;14:989-994.

14. Gray HW, McKillop JH, Bessent RG. Lung scan reporting language: what does it mean? Nucl Med Commun. 1993;14:1084-1087.

15. Value of the ventilation/perfusion scan in acute pulmonary embolism: results of the prospective investigation of pulmonary embolism diagnosis (PIOPED). The PIOPED Investigators. JAMA. 1990;263:2753-2759.

16. Stein PD, Fowler SE, Goodman LR, et al. Multidetector computed tomography for acute pulmonary embolism. N Engl J Med. 2006;354:2317-2327.

17. Stein PD, Woodard PK, Weg JG, et al. Diagnostic pathways in acute pulmonary embolism: recommendations of the PIOPED II investigators. Am J Med. 2006; 119:1048-1055.

18. Stein EG, Haramati LB, Chamarthy M, et al. Success of a safe and simple algorithm to reduce use of CT pulmonary angiography in the emergency department. AJR. 2010;194:392-397.

19. Gottschalk A, Sostman HD, Coleman RE, et al. Ventilation-perfusion scintigraphy in the PIOPED study. Part II. Evaluation of the scintigraphic criteria and interpretations. J Nucl Med. 1993;34:1119-1126.

20. Stein PD, Gottschalk A, Henry JW, et al. Stratification of patients according to prior cardiopulmonary disease and probability assessment based on the number of mismatched segmental equivalent perfusion defects: approaches to strengthen the diagnostic value of ventilation/perfusion lung scans in acute pulmonary embolism. Chest. 1993;104:1461-1467.

21. Anderson DR, Kovacs MJ, Dennie C, et al. Use of spiral computed tomography contrast angiography and ultrasonography to exclude the diagnosis of pulmonary embolism in the emergency department. J Emerg Med. 2005;29:399-404.

22. Analyze a $2 \times 2$ contingency table. GraphPad Software Web site. Available at: http://graphpad.com/quickcalcs/contingency1.cfm. Accessed July 11, 2011.

23. Reinartz P, Wildberger JE, Schaefer W, et al. Tomographic imaging in the diagnosis of pulmonary embolism: a comparison between V/Q lung scintigraphy in SPECT technique and multislice spiral CT. J Nucl Med. 2004;45: 1501-1508.

24. Leblanc M, Leveillee F, Turcotte E. Prospective evaluation of the negative predictive value of V/Q SPECT using 99mTc-Technegas. Nucl Med Commun. 2007;28:667-672.

25. Stein PD, Freeman LM, Sostman HD, et al. SPECT in acute pulmonary embolism. J Nucl Med. 2009;50:1999-2007.

26. Freeman LM. Don't bury the V/Q scan: it's as good as multidetector CT angiograms with a lot less radiation exposure. J Nucl Med. 2008;49:5-8.

27. Freeman LM, Haramati LB. V/Q scintigraphy: alive, well and equal to the challenge of CT angiography. Eur J Nucl Med Mol Imaging. 2009;36:499-504. 